\title{
Theorie in der Soziologie ${ }^{1}$
}

\section{Von Norman Braun}

Zusammenfassung: Die soziologische Theorie wird u.a. aufgrund ihrer oftmals bestehenden Unklarheit, ihrer häufigen Beschränktheit auf Beschreibungen und Kategorisierungen, der Unvereinbarkeit ihrer vielfältigen Perspektiven und ihrer überwiegend praktizierten Vernachlässigung der Empirie nicht nur von anderen Fächern, sondern auch von der wissenschaftlich interessierten Öffentlichkeit weitgehend ignoriert. Ihre Rehabilitation erfordert, dass sich Theorieauffassung und Theoriebildung in der Soziologie an den einschlägigen Vorstellungen erfolgreicher empirischer Wissenschaften orientieren. Damit ist eine Theoriekonzeption zu übernehmen, die in der quantitativ orientierten empirischen Sozialforschung verbreitet ist. Eine Theorie ist danach eine Menge von Aussagen, die durch deduktive Argumentationsketten verknüpft sind und sich zumindest teilweise empirisch überprüfen lassen. Diese Theorieauffassung und ihre Hintergründe werden dargestellt.

\section{Einführung}

Empirische Wissenschaft beruht auf der Vorstellung, dass Zusammenhänge im jeweils untersuchten Bereich auftreten, die erkannt und begründet werden können. Forscher nehmen an, dass verstehbar ist, warum etwas passiert. Ihre Antworten auf diese Fragen sind Erklärungen. Im Idealfall sind dadurch die wesentlichen notwendigen und hinreichenden Bedingungen für das Auftreten von bestimmten Ereignissen zu einem gewissen Zeitpunkt, von Veränderungen im Zeitverlauf und von Zusammenhängen von Zuständen und Ereignissen in Raum und Zeit spezifiziert. Anders gesagt: Im denkbar besten Szenario sind, unabhängig vom jeweiligen Fach, sämtliche relevanten Voraussetzungen für die fraglichen Sachverhalte und Abläufe identifiziert und in kausalen Beziehungen miteinander verknüpft, wodurch festgelegt wird, wann etwas wo stattfindet oder abläuft. In einem weniger idealisierten Fall kennt man nicht alle zentralen Bedingungen und / oder Kausalbeziehungen, weshalb man dann auch kein umfassendes Wissen über das Eintreten bestimmter Phänomene und Prozesse besitzt und Prognosen darüber entsprechend erschwert sind.

Erklärungen lassen sich verallgemeinern und schlagen sich in Theorien nieder, die ihrerseits eventuell Vorhersagen über Geschehnisse oder Verläufe erlauben. In allen empirischen Wissenschaften müssten die Erstellung, Prüfung und Verbesserung von solchen erklärenden Theorien als zentrale Aktivitäten gelten. Neben den Naturwissenschaften sollten also auch die Sozialwissenschaften an erklärenden Theorien, deren empirischen Tests und ihren etwaigen Weiterentwicklungen interessiert sein.

Obwohl sie von ihrem Selbstverständnis her eine empirische Wissenschaft ist, besitzt die Soziologie kein derartiges einheitliches Verständnis von Theorie und deren Zweck. Vielmehr existieren in der Soziologie unterschiedlichste Theorieauffassungen und Theorieansätze, was die Relevanz und Popularität soziologischer Überlegungen in anderen Fächern und der wissenschaftlich interessierten Öffentlichkeit nicht gerade erhöht. Im Gefolge von z.B. Albert (1964), Bunge (1996) oder Opp (2005) plädiert der vorliegende Aufsatz daher für die soziologische Übernahme der skizzierten Auffassung von Theorie, die in praktisch allen führenden Feldern der Forschung verwendet wird. Diese Konzeption wird in der quantitativ ori-

1) Für hilfreiche Diskussionen, Hinweise und Verbesserungsvorschläge ist Roger Berger, Christian Ganser, Jochen Groß, Marc Keuschnigg, Eva Negele, Thomas Wimmer und Tobias Wolbring zu danken. 
entierten Sozialforschung (z.B. Diekmann 2007) bereits vertreten und u.a. im Rahmen des Rational-Choice-Ansatzes (z.B. Coleman 1990; Esser 1993) und der analytischen Soziologie (Hedström 2005) akzeptiert.

Der übrige Aufsatz ist wie folgt gegliedert: Zunächst werden einige der mit der bestehenden Theorienvielfalt in der Soziologie einhergehenden Probleme kurz beschrieben (Abschnitt 2). Dann wird die Notwendigkeit von Erklärungen betont und es werden Erklärungsvarianten angeführt, die dabei als Orientierungshilfen dienen können (Abschnitt 3). Es folgt eine Festlegung des Theoriebegriffs, eine Erörterung von Prinzipien der Theoriebildung und ihren Folgen sowie eine Illustration der hier vertretenen Theorieauffassung durch ein Beispiel (Abschnitt 4). Danach wird diskutiert, inwieweit theoretische Annahmen wirklichkeitsnah zu spezifizieren sind (Abschnitt 5). Schließlich werden Kriterien für den Vergleich konkurrierender Theorien referiert und Aspekte der Wissensentwicklung besprochen (Abschnitt $6)$.

\section{Theoriepluralismus in der Soziologie}

In der Soziologie existiert eine bemerkenswerte Theorienvielfalt. Gleichzeitig gibt es theoretische Perspektiven im Fach, die weitgehend unvereinbar sind. Soziologische Forschungsansätze stehen dabei kaum im Wettbewerb, sondern werden nebeneinander ziemlich konfliktfrei verfolgt. Der Pluralismus von Denktraditionen und Theorien wird dabei oft als eine Stärke des Faches gedeutet. Allerdings stellt diese Heterogenität sicherlich einen wesentlichen Grund für die Schwierigkeit dar, einen Wissensbestand der Soziologie zu identifizieren, der zumindest in der Disziplin mehrheitlich akzeptiert wird. Abgesehen von den Arbeiten einiger Klassiker kann sich die Mehrheit der Soziologen vermutlich nicht auf diejenigen sozialtheoretischen Erkenntnisse einigen, die über die Grenzen des Faches hinaus kommuniziert werden sollten.

Dabei ist der Anspruch vieler soziologischer Theoretiker alles andere als bescheiden. Oftmals handelt es sich bei den jeweiligen Beiträgen nämlich keineswegs um Theorien mittlerer Reichweite im Sinne von Merton (1957), sondern eher um Großtheorien mit sehr breiten Geltungsansprüchen. Als soziologische Theorien werden u.a. philosophisch angehauchte Analyserahmen und Weltanschauungen, eigenwillige Beschreibungen und Deutungen, mehr oder weniger erschöpfende Kategorisierungen und Begrifflichkeiten sowie journalistisch anmutende Zeitdiagnosen bezeichnet. Nach Joas / Knöbl (2004: 17) besteht lediglich Übereinstimmung darüber, dass Theorien als generalisierende Aussagen aufgefasst werden können. Die damit einhergehende Akzeptanz von fast allen denkbaren Theorieauffassungen hat wohl auch dazu geführt, dass nahezu alle aktuellen deutschsprachigen Lehrbücher der theoretischen Soziologie auf wissenschaftstheoretische Erörterungen verzichten.

Im Regelfall sind die jeweiligen Theorien zudem rein verbaler Natur. Theoriebildung erfolgt in der Soziologie auch heutzutage fast immer ohne Logik und Mathematik. Während andere Disziplinen bei Theoriekonstruktionen in zunehmender Weise formale Hilfsmittel z.B. für Deduktionen, Gedankenexperimente und Computersimulationen in Anspruch nehmen, wird in der Soziologie bei der theoretischen Arbeit üblicherweise noch wie vor Jahrhunderten vorgegangen. Deduktiv gewonnene Erkenntnisse z.B. aus Nachbarfächern werden in der soziologischen Theoriediskussion nahezu ignoriert, obwohl gerade die Formalisierung eine Disziplingrenzen übergreifende Verständlichkeit gewährleistet. Wahrscheinlich reflektiert dies, dass praktisch kein Wissen über die jeweils einschlägigen Verfahren und Techniken vorhanden ist. In der Soziologie-Ausbildung werden derartige Kenntnisse nämlich fast nirgendwo vermittelt. Freiwillig beschäftigt man sich wohl kaum mit dem anstrengenden Ausbau der eigenen analytischen Fertigkeiten, wenn keine entsprechenden Anreize bestehen. 
Tatsächlich orientiert sich die Selektion des Theoretikernachwuchses ja nicht an logisch-mathematischen Fähigkeiten, sondern eher an rhetorisch-sprachlichen Kompetenzen.

Daraus resultierende negative Konsequenzen für die Theoriebildung sind normalerweise beeindruckend weitschweifige, häufig aber wenig präzise theoretische Ausführungen, die zudem oft durch schwierig zu identifizierende Annahmen und Folgerungen, schwammige Konzepte und Begrifflichkeiten sowie unschlüssige oder gar fehlerhafte Argumentationen gekennzeichnet sind. Derartige Mängel haben vermutlich dazu beigetragen, dass sich einzelne soziologische Theorierichtungen verselbständigt haben und sich jeweils stark mit sich selbst beschäftigen - Insider schreiben für Insider über Detailprobleme, die keinen Outsider ernsthaft beschäftigen würden.

Die vielfältigen Unklarheiten sind im Übrigen mit dafür verantwortlich, was Calhoun (1996) zu Recht beklagt: Soziologische Theorie besteht seit geraumer Zeit auch darin, vertiefende Interpretationen und Klarstellungen von Aussagen bereits toter Soziologen anzufertigen oder sich über derartige Arbeiten kritisch zu äußern, die andere Autoren über das Werk bereits toter Soziologen vorgelegt haben. Dies dürfte auch mit der universitären Lehrpraxis der soziologischen Theorie zu tun haben: Wie Coleman (1990) feststellt und ein Blick in aktuelle Lehrbücher bestätigt, ist die unterrichtete Sozialtheorie eine Geschichte sozialwissenschaftlicher Ideen der letzten Jahrhunderte. Ihr Schwerpunkt liegt damit keineswegs auf den empirisch hinreichend gesicherten und für die heutigen Gesellschaften relevanten Einsichten. Vielmehr werden die Gedanken und Konzepte von als wichtig erachteten Sozialtheoretikern hintereinander und / oder teilweise vergleichend nacherzählt und durch die Lektüre entsprechender Texte gestützt. Nimmt man nun an, dass die Ideen z.B. der Klassiker der Soziologie heutzutage kaum mehr praktische Bedeutung haben, dann stellt sich die Frage nach dem Sinn eines derartigen Unterrichts; nimmt man dagegen an, dass diese Ideen auch heute noch Relevanz besitzen, dann ist unverständlich, warum die Existenz gesetzesähnlicher Aussagen durch soziologische Theoretiker normalerweise bezweifelt wird.

In erklärenden Theorien spielen solche robusten Hypothesen eine wesentliche Rolle. Allerdings verzichten soziologische Theoretiker überwiegend darauf, überhaupt Erklärungsprobleme zu spezifizieren. Alternativ ausgedrückt: Im Gegensatz zu Beschreibungen und Typologien stehen Erklärungen typischerweise nicht im Zentrum der soziologischen Theoriebildung. Wie Hedström (2005: 13) betont, beziehen sich Beschreibungen und Typologien aber auf andere Fragen als Erklärungen. Während die Erklärung die Frage nach Gründen beantwortet, gibt die Beschreibung an, was der Fall ist und die Typologie legt fest, welche Kategorien zu unterscheiden sind. Selbst wenn Beschreibungen und Typologien jeweils Grundlagen für Erklärungen schaffen, können sie diese keinesfalls ersetzen.

Auch aufgrund des Mangels an soziologischen Erklärungen verwundert es nicht, dass die soziologische Theorie in der wissenschaftlich interessierten Öffentlichkeit praktisch kaum mehr eine Rolle spielt. Wenn Soziologie in der Öffentlichkeit überhaupt gefragt ist, dann bezieht sich diese Nachfrage üblicherweise auf hinreichend verallgemeinerungsfähige Befunde der empirischen Sozialforschung, jedoch kaum auf ihre theoretischen Einsichten. Damit besteht ein wesentlicher Unterschied beispielsweise zur Ökonomik, deren theoretische Erkenntnisse immer wieder Gegenstand öffentlicher Debatten sind.

Vor dem skizzierten Hintergrund ist zudem wenig überraschend, dass die soziologische Theorie von eher formal orientierten Nachbarfächern wie etwa der Ökonomik und der amerikanischen Politikwissenschaft selbst dann weitgehend ignoriert wird, wenn sich diese mit genuin soziologischen Themen (wie etwa der Befolgung sozialer Normen, der Entstehung sozialer Ordnung oder der Etablierung und Verfestigung von Statushierarchien) beschäftigen. Im Gegensatz zu den Erkenntnissen dieser Disziplinen scheinen im Übrigen selbst lange vorliegende soziologische Theorien nur wenig praktische Relevanz zu haben, weil sie offen- 
bar kaum Implikationen für die Gestaltung von z.B. Institutionen, Organisationen und Strukturen mit sich bringen.

Die heutige weitgehende Irrelevanz der soziologischen Theorie hat sicher auch mit der recht strikten Trennung zwischen Theorie und Empirie im Fach zu tun. Dies beginnt bereits in der Ausbildung: In keinem der führenden deutschsprachigen Theorielehrbücher (Joas / Knöbl 2004; Mikl-Horke 2001; Münch 2002; 2003; 2004; Rosa / Strecker / Kottmann 2007) finden sich systematisch aufbereitete Informationen über eventuell vorhandene empirische Befunde zu den referierten Theorien. Vielmehr wird in den meisten Fällen sogar verschwiegen, dass die vorgestellten Theorien eigentlich keine testbaren Annahmen und Folgerungen haben oder, falls solche prüfbaren Aussagen vorliegen, bisher anscheinend auf empirische Überprüfungen verzichtet wurde.

Obwohl die Soziologie auch innerhalb des Faches als empirische Wissenschaft betrachtet wird, schlägt sich dies bei der Theoriebildung leider kaum nieder. So werden bei der Theoriekonstruktion gelegentlich etwaige empirische Erkenntnisse auf falsche oder nur auf sehr selektive Weise berichtet. Zumeist aber werden empirische Befunde entweder gar nicht zur Kenntnis genommen oder einfach als irrelevant abgetan. Ähnliches gilt im Regelfall auch für einschlägige Arbeiten aus Nachbardisziplinen (wie z.B. Evolutionäre Anthropologie, Entscheidungs- und Spieltheorie, Evolutionäre Psychologie, Experimentelle Verhaltensforschung, Mikroökonomik und Sozialpsychologie).

Soziologische Theoretiker kümmern sich überdies nur selten um die empirische Prüfbarkeit von wenigstens einer Teilmenge ihrer Ausführungen. Häufig sind die jeweiligen Aussagen nicht einmal präzise genug formuliert, um überhaupt empirisch falsch sein zu können. Nur in seltenen Fällen wird überdies durch die Theorie vorgegeben, wie ein bestimmtes Konzept in einer empirischen Untersuchung gemessen werden soll. Besteht überhaupt irgendein empirischer Anspruch des jeweiligen Theoretikers, so wird dieser nicht selten mit Verweisen auf schwerlich verallgemeinerungsfähige qualitative Fallstudien oder Beispiele aus der Alltagserfahrung und der Medienberichterstattung eher schlecht als recht eingelöst.

Insgesamt kann man der soziologischen Theorie also ihre oftmals bestehende Unklarheit, ihre häufige Beschränktheit auf Beschreibungen und Kategorisierungen, die Unvereinbarkeit ihrer vielfältigen Perspektiven und ihre überwiegend praktizierte Vernachlässigung der Empirie vorwerfen. Insbesondere aus diesen Gründen wird sie wohl nicht nur von anderen Fächern, sondern auch von der wissenschaftlich interessierten Öffentlichkeit weitgehend ignoriert. Vermutlich würde bereits die regelmäßige Berücksichtigung empirischer Evidenz bei der Theoriebildung, die Konzentration auf Erklärungen und die Gewährleistung der systematischen Überprüfbarkeit von theoretischen Aussagen die Außenwirkung, Klarheit und Relevanz der soziologischen Theorie verbessern.

Im Gegensatz zu vielen Soziologen mit einer Ausrichtung an der qualitativen Sozialforschung und ihren vielfältigen Ausprägungen sind sich quantitativ orientierte Sozialforscher über diese Punkte recht einig. Sie stimmen darüber hinaus in ihren Auffassungen über die Theoriebildung weitgehend überein. Daneben bestehen zwischen der von ihnen vertretenen Konzeption einer erklärenden Theorie und den metatheoretischen Vorstellungen von anderen empirisch orientierten Wissenschaftlern (z.B. aus der Anthropologie, Betriebswirtschaftslehre, Biologie, Ökonomik und Politologie) erheblich weniger Konfliktpunkte als mit ihren eher qualitativ orientierten Fachkollegen. Zudem korrespondiert die damit einhergehende Theorieauffassung mit der analytischen Soziologie, die insbesondere Hedström (2005) propagiert und dem Rational-Choice-Ansatz (z.B. Diekmann und Voss 2004), der in verschiedenen sozialwissenschaftlichen Disziplinen weltweit Verwendung findet. $\mathrm{Zu}$ betonen ist freilich, dass sie nicht an die analytische Soziologie und den Rational-Choice-Ansatz ge- 
bunden ist. Dies wird bereits deutlich, wenn man sich mit den Grundlagen und Zielsetzungen dieser Theorieauffassung beschäftigt.

\section{Vororientierungen und Erklärungen}

Jede wissenschaftliche Analyse erfordert eine widerspruchslose Argumentation. Theoriekonzeption und Theorieentwicklung werden daneben von anderen Voraussetzungen geprägt. Es ist sinnvoll, die Darstellung mit nicht weiter hinterfragten Postulaten zu beginnen.

\subsection{Grundlegende Prämissen}

Quantitativ orientierte empirische Sozialforscher sind üblicherweise keine Konstruktivisten, sondern Realisten. Für sie ist die Wirklichkeit demnach nicht nur eine Vorstellung, in der man sich alle Dinge und Vorkommnisse letztlich selber schafft. Folgende Annahmen werden stattdessen vorausgesetzt:

a. Materielle Objekte (z.B. chemische Substanzen, Pflanzen), natürliche Ereignisse (z.B. Geburt, Tod) und Abläufe (z.B. Altern, biochemische Prozesse) sowie soziale Phänomene (z.B. Kooperation, Konflikt) und Prozesse (z.B. Ausbreitung von Neuerungen, Etablierung von Traditionen) existieren prinzipiell autonom von menschlichen Sinneserfahrungen und Deutungsversuchen. Entgegen verschiedener idealistisch geprägter Denktraditionen (wie etwa Dekonstruktivismus, Phänomenologie, Semiotik, Strukturalismus und Poststrukturalismus) wird die Wirklichkeit demnach nicht im Diskurs geschaffen. Vielmehr gibt es eine einzige Realität, die unabhängig von Worten und Zeichen ist. Ihre Existenz wird postuliert, obwohl dafür genauso wenig wie für das Gegenteil (d.h. Abwesenheit einer objektiven Realität, aber Vorliegen subjektiv konstruierter Wirklichkeiten) ein Beweis geführt werden kann.

b. Es existieren Zusammenhänge in der Wirklichkeit, die durch Menschen vor dem Hintergrund soziokulturell vorgeformter Bezüge erkannt und verstanden werden können. Erkenntnis und Verständnis sind jeweils Annäherungen an die Wahrheit, die selbst nicht auf Konventionen beruht. Es lassen sich also Theorien und damit Hypothesen spezifizieren, die sich zumindest teilweise empirisch prüfen lassen und bestenfalls eine weitgehende Korrespondenz mit der Wahrheit aufweisen. Die Wirklichkeit gibt die „Grundlinie“ für die Theoriebildung vor - irgendein empirischer Bezug ist auch dann unverzichtbar, wenn man die Notwendigkeit von vielfältigen Abstraktionen in ihrem Rahmen einsieht. Vor der theoretischen Arbeit sind daher empirische Erkenntnisse zur Kenntnis zu nehmen, bei etwaigen empirischen Untersuchungen sind auch theoretische Prämissen ohne Test zu unterstellen.

c. Konzepte und Theorien zu beliebigen Sachverhalten und Abläufen stellen menschliche Konstruktionen dar. Für das Aufdecken und Begreifen von Zusammenhängen und die Festlegung von Konzepten ist Vernunft notwendig, wenn auch nicht hinreichend. Menschen entscheiden und handeln aus Gründen, weswegen Entscheidungen und Handlungen typischerweise als zielgerichtet aufzufassen sind. Vernunft schlägt sich jeweils in prinzipiell situationsgerechten Entscheidungen und Handlungen nieder. Entscheidungen und Handlungen zu verstehen, bedeutet daher, sie als vernünftig im Sinne der Situationserfordernisse und daher als zweckdienlich zu erkennen.

d. Bei der Theoriebildung ist die Berücksichtigung des einschlägigen verfügbaren Wissens vor dem Hintergrund der situativen Gegebenheiten zweckmäßig. Wissen über die Realität entsteht in und verändert sich mit der Erfahrung durch Wahrnehmung und Lernen. Schon deshalb erscheint die ständige Konfrontation theoretischer Hypothesen mit empirischen Daten unverzichtbar. Theorien bleiben vorläufiges Wissen, da sie durch empirische Evidenz immer in Frage gestellt werden können. Es gibt kein Verfahren, Theorien zur letztendlichen Wahrheit zu erheben. 
Auch deshalb entspricht die hier vertretene Sichtweise in wesentlichen Punkten dem von Karl Popper (1934 [1994]; 1944 [1987]; 1945a, b [1992 a, b]; 1963 [1994]) geprägten kritischen Rationalismus, der von vielen Empirikern akzeptiert wird. Soziologisch relevante Sachverhalte und Prozesse betreffen vor diesem Hintergrund keine zwingend subjektiven Konstrukte, sondern möglichst objektiv zu erfassende Entitäten. So sind etwa die Verteilungen von Begabungen, Körpermerkmalen und Wohlstandsniveaus in den jeweils betrachteten Populationen und deren Wirkungen keineswegs nur subjektiv erfahrbar - beispielsweise führen objektiv nachweisbare Entlohnungsdifferenzen zu realen Kaufkraftunterschieden zwischen prinzipiell vergleichbaren Frauen und Männern im deutschen Erwerbsleben. Aufgrund der damit bestehenden Parallelen zu anderen empirischen Disziplinen ist nach der Rolle von Erklärungen zu fragen.

\subsection{Erklärung als Erfordernis}

Zwischen Natur und Gesellschaft gibt es fundamentale Unterschiede, die sich in den jeweiligen Wissenschaften auch niederschlagen. Beispielsweise erscheint es in den Naturwissenschaften wenig problematisch, bei einer Argumentation im Sinne eines kausalen Zusammenhangs davon auszugehen, dass eine Ursache immer vor der Wirkung eintritt. Aufgrund der menschlichen Fähigkeit der Antizipation ist eine solche Abfolge von Ursache und Wirkung allerdings bei einem etwaigen kausalen Zusammenhang in den Sozialwissenschaften keineswegs immer gegeben (z.B. wird es nicht deshalb Sommer, weil man sich im Frühjahr eine Badehose gekauft hat).

Eine weitere Illustration eines grundlegenden Unterschiedes zwischen den Natur- und Sozialwissenschaften betrifft die potenziellen Wirkungen von Vorhersagen. Im Gegensatz zu den Naturwissenschaften kann eine Prognose in den Sozialwissenschaften eine Eigendynamik entwickeln und allein deshalb unbeabsichtigte Folgen haben. Nach Robert Mertons (1936) klassischem Beitrag kann es sich dabei nicht nur um eine sich selbst erfüllende Prognose (,Self-Fulfilling Prophecy“) wie z.B. eine Bankenpleite aufgrund entsprechender Gerüchte, sondern auch um eine sich selbst zerstörende Vorhersage (,Self-Destroying Prophecy") handeln (z.B. durch gegenläufige Reaktionen von Spekulanten auf Wirtschaftsprognosen).

Man kann das angesprochene Problem der unbeabsichtigten Vorhersagedynamik wie auch die zuvor diskutierte Problemstellung der eventuell unsicheren Abfolge von Ursache und Wirkung als Besonderheiten der Sozialwissenschaften ansehen und u.a. deshalb eine eigene Methodologie dieser Disziplinen fordern. Angemessener erscheint jedoch, sie in Übereinstimmung mit Nachbardisziplinen wie etwa der Anthropologie, Betriebswirtschaftslehre, Ökonomik, Politologie und Sozialpsychologie als zusätzliche Erschwernisse zu betrachten, die sich, neben den für alle empirischen Wissenschaften relevanten Problemen, insbesondere in den Sozialwissenschaften stellen. Hintergrund dieser Auffassung ist, dass sich die grundlegenden Aufgaben empirischer Wissenschaften ähneln: Sie bestehen in Beschreibung (Was ist der Fall?), Erklärung (Warum ist etwas der Fall?) und Vorhersage (Was wird der Fall sein?).

Trotz ihrer Unterschiedlichkeit sind Beschreibungen und Erklärungen verknüpft - eine Beschreibung eines Sachverhaltes oder Ablaufs kann Ausgangspunkt für seine Erklärung sein. Erklärungen liefern kausale Aussagen, die Vorhersagen erlauben. Prognosen beruhen deshalb oft auf Erklärungen.

Die modernen Naturwissenschaften präsentieren Theorien, die viele natürliche Phänomene und Prozesse ursächlich begründen und daher empirisch prüfbare Vorhersagen über Sachverhalte und Abläufe in der Natur gestatten. Die Sozialwissenschaften sind in der Natur verwurzelt, ohne Teil der Naturwissenschaften zu sein. Jedes Individuum unterliegt natürlichen 
und sozialen Einflüssen, jede Gesellschaft ist auch durch die Natur geprägt. Beispielsweise hat die naturwissenschaftliche Tatsache der Sterblichkeit von Menschen eine Vielzahl von nicht unbedingt deckungsgleichen Auswirkungen auf Hierarchien, Institutionen und Traditionen in verschiedenen Gesellschaften. Weiter haben z.B. das Klima und die dadurch gegebenen Möglichkeiten und Einschränkungen wichtige Konsequenzen für das menschliche Zusammenleben und Wirtschaften. Zudem gibt es eine ganze Reihe von wissenschaftlichen Hybriddisziplinen wie etwa Bioökonomik, Demographie, Epidemiologie, Geographie, Gerontologie und Psychophysik. Die Existenz dieser Hybridformen zeigt deutlich, dass eine strikte Trennung von Naturwissenschaften und Sozialwissenschaften nicht durchgehalten werden kann.

Bedenkt man überdies die v.a. durch naturwissenschaftliche Erkenntnisse erreichte Verbesserung und Verlängerung des menschlichen Lebens, so liegt die Forderung nahe, sich bei der Bearbeitung sozialwissenschaftlicher Themenstellungen stärker als bisher am relativ fruchtbaren Vorgehen in den Naturwissenschaften zu orientieren. Damit ist allerdings keineswegs die unkritische Übernahme eines naturwissenschaftlich geprägten Weltbilds gemeint. Die Forderung bedeutet vielmehr, Erklärungen auch in den Sozialwissenschaften als Zielsetzungen anzusehen. Es genügt demnach nicht, möglichst genaue Rekonstruktionen sozialer Phänomene und Prozesse auf der Grundlage von Sinnzuschreibungen und Sinndeutungen etwa in der Tradition der interpretativen Soziologie anzufertigen. Als wesentlich erscheinen vor diesem Hintergrund (und in Übereinstimmung mit Max Webers bekannten Vorstellungen) nämlich auch in der Soziologie jeweils Antworten auf Warum-Fragen. Trotz Publikationen, welche bereits vor Jahrzehnten diese Perspektive eingenommen haben (siehe z.B. Brodbeck 1969), wird die Möglichkeit und Notwendigkeit von Erklärungen aber zumindest in der Soziologie nach wie vor bezweifelt (u.a. Ethnomethodologie, verstehende Soziologie). Anders gesagt: Keineswegs alle Soziologen erheben einen Erklärungsanspruch bei der Theoriebildung. Eine genauere Beschäftigung mit Erklärungen erscheint daher angebracht.

\subsection{Wissenschaftliche Erklärungen}

Die Vorgehensweise bei der Beantwortung von Warum-Fragen in den Sozialwissenschaften kann sich prinzipiell an die Praxis der Naturwissenschaften anlehnen. Im Rahmen einer Erklärung erfolgen dort üblicherweise kausale Aussagen. In der Soziologie (z.B. Esser 1993) wird in diesem Zusammenhang typischerweise empfohlen, sich am Ideal der deduktiv-nomologischen Erklärungssystematik von Carl Hempel und Paul Oppenheim (1948) zu orientieren, bei denen der zu erklärende Sachverhalt (Explanandum) aus einer wahren Prämissenmenge (Explanans) logisch (nämlich im Sinne des „modus ponens“) abgeleitet wird. Dabei muss das Explanans wenigstens zwei Komponenten enthalten, nämlich die faktisch korrekten Anfangs- oder Randbedingungen und zumindest eine empirisch zutreffende nomologische Hypothese (Gesetzmäßigkeit). Letztere ist deterministisch und gibt an, wie aus dem Vorliegen bestimmter Anfangs- oder Randbedingungen das Explanandum folgt, welches seinerseits in möglichst genauer Beschreibung vorliegen sollte.

Allerdings existieren Grenzen dieses Erklärungsschemas, die z.T. bereits von Hempel (1965) thematisiert wurden. Selbst wenn alle Voraussetzungen für eine deduktiv-nomologische Erklärung gegeben sind, gelingt nämlich keineswegs immer eine überzeugende Erklärung im Rahmen des Schemas. Will man beispielsweise erklären, warum eine sexuell enthaltsame Frau bisher nicht schwanger wurde, so kann man hierfür die empirisch beobachtete Anfangsbedingung einer regelmäßigen Einnahme der Anti-Baby-Pille durch diese Frau mit der empirisch zutreffenden Gesetzmäßigkeit kombinieren, wonach Schwangerschaften durch die regelmäßige Einnahme der Anti-Baby-Pille verhindert werden. Freilich liegt damit keine 
korrekte Erklärung vor, obwohl die Anforderungen des Hempel-Oppenheim-Schemas erfüllt sind - die Erklärung überzeugt nicht, weil sie auf den falschen kausalen Zusammenhang verweist.

Aufgrund solcher Schwächen kann man das Hempel-Oppenheim-Modell bestenfalls als Ausgangspunkt und erste Orientierungshilfe für die Lösung des Problems der Erklärung ansehen. Nicht übersehen sollte man insbesondere die letzten Jahrzehnte der wissenschaftstheoretischen Diskussion des Erklärungskonzeptes, die von Salmon (2006) referiert wird. Besonders interessant erscheinen hierbei Ideen zur Kausalität, die von dem Informatiker und Statistiker Judea Pearl (2000) stammen. Unter Verwendung seiner Einsichten präsentiert der Philosoph James Woodward (2003) eine Theorie der kausalen Erklärung. Stephen Morgan und Christopher Winship (2007) zeigen, dass diese Theorie mit methodischen Überlegungen zu Fragen des Forschungsdesigns und der Datenanalyse in den Sozialwissenschaften korrespondiert.

Daneben weisen sie auch auf die gleichfalls vorhandene Kompatibilität ihrer methodischen Vorstellungen mit der Idee der Mechanismus-basierten Erklärung hin, die trotz ihrer Verbreitung in der sozialwissenschaftlichen Forschungspraxis (siehe die Beiträge in Hedström / Swedberg 1998) erst in jüngerer Zeit verstärkt diskutiert wird (Hedström 2005; Mayntz 2004; Schmid 2006). Sie bietet eine alternative Orientierungshilfe, wenn man eine Erklärung sozialer Sachverhalte oder Abläufe versucht. Eine Mechanismus-basierte Erklärung eines beobachteten Phänomens oder Prozesses liegt dann vor, wenn auf den kausalen Mechanismus verwiesen wird, der in dem System unter Betrachtung regelmäßig zu einem solchen Sachverhalt oder Ablauf führt. In der Soziologie stellen Mechanismus-basierte Erklärungen also auf soziale Mechanismen wie z.B. Homophilie, Imitation, Konkurrenz oder Vakanzketten ab, um wahrscheinliche soziale Konsequenzen aus Interaktionen von Akteuren unter gegebenen sozialen Bedingungen zu identifizieren.

Eine mit den diskutierten Idealbildern der Erklärung prinzipiell vereinbare Variante ergibt sich bei soziologischen Ansätzen, die Theoriebildung im Sinne des „methodologischen Individualismus“" präferieren (u.a. Boudon 1987; Coleman 1990; Raub / Voss 1981; Wippler / Lindenberg 1987). Nach übereinstimmender Auffassung sind Soziologen vorwiegend an der Erklärung von Makrophänomenen (z.B. Prävalenz und Entwicklung des Drogengebrauchs in der Gesellschaft, Veränderungen von Familiengrößen und Scheidungsraten) interessiert. Daraus kann nun aber nicht geschlossen werden, dass sich die Erklärung auf Zusammenhänge der Makroebene beschränken muss. Vielmehr kann man das soziale Geschehen durchaus als Resultat der Verflechtung individueller Handlungen begreifen, also die Mikroebene der Individuen bei der soziologischen Analyse berücksichtigen. Verdeutlicht wurde dies durch frühe Untersuchungen sozialer Märkte und Austauschbeziehungen. Bekannte Beispiele sind Homans (1974) behavioristisch fundierte Tauschtheorie, Emersons (1962) Beitrag zu Macht und Abhängigkeit in dyadischen Beziehungen, Blaus (1964) strukturelle Analyse sozialer Tauschvorgänge sowie Colemans (1973) Modell zum Stimmentausch in legislativen Körperschaften.

Nach Vanberg (1975) verhinderte allerdings das Selbstverständnis der Soziologie als eigenständige wissenschaftliche Disziplin die allgemeine Akzeptanz des (auf Moralphilosophen wie z.B. David Hume oder Adam Smith zurückreichenden) individualistischen Ansatzes. Das Bedürfnis nach Abgrenzung führte vielmehr zu einer relativen Dominanz „spezifisch soziologischer“ Theorien, welche nur die Makroebene des betrachteten Sozialsystems betonten. Nicht nur nach Vanbergs Einschätzung erwies sich diese „kollektivistische Soziologie" jedoch als wenig fruchtbar. Lindenberg (1996) hebt in diesem Zusammenhang hervor, dass sich vermeintlich robuste Makroaussagen regelmäßig als abhängig von Raum und Zeit erwiesen. 
In den letzten Jahrzehnten mehrten sich deswegen Stimmen (z.B. Boudon 1980; Coleman 1990; Esser 1993; Raub / Voss 1981; Wippler / Lindenberg 1987), die für die Spezifikation von hinreichend allgemeinen Aussagen auf der Mikroebene der Akteure (zumeist Individuen, gelegentlich aber auch Haushalte, Firmen oder Bürokratien) in soziologischen Erklärungen plädieren. Danach garantiert die Konstanz der menschlichen Natur eine gewisse Stabilität der Verhaltensweisen, was man bei der Erklärung von Makrozusammenhängen im Sinne des methodologischen Individualismus berücksichtigen kann.

Eine andere Begründung für die Einbeziehung der Mikroebene der Entscheidungsträger in soziologischen Erklärungen ergibt sich, wenn man Sozialtheorie mit z.B. Joas / Knöbl (2004) ausgehend von folgenden Prämissen betreiben will: Menschliche Handlungen erfolgen aus Gründen; aus dem menschlichen Zusammenleben bilden sich Ordnungen; Ordnungen wandeln sich in der Zeit. Um diese abstrakten Überlegungen zu konkretisieren, bedarf es eines geeigneten handlungstheoretischen Ansatzes.

Nach Lindenberg (1996) sollte ein solcher Ansatz so beschaffen sein, dass er ohne die Sammlung enormer Datenmengen für jedes Individuum auskommt und somit bei der Analyse soziologischer Fragen relativ problemlos anwendbar ist. Es verwundert deswegen nicht, dass viele individualistische Soziologen dem Vorschlag Poppers (1985 [1995]) folgen und mit dem „Rationalitätsprinzip“ voraussetzen, dass Akteure jeweils situationsangemessen agieren. Dieses Prinzip ist dabei nicht als empirisch bestätigte Gesetzmäßigkeit oder als psychologisches Postulat zu verstehen. Es stellt vielmehr eine methodologische Idealisierung dar, mit dessen Hilfe menschliche Handlungen und situative Gegebenheiten miteinander verknüpft werden. Zur Verdeutlichung kann man die Schritte einer situationsbezogenen Handlungserklärung anführen, die von der Philosophin Noretta Koertge (1975: 440) zur Klarstellung der Popperschen Ideen spezifiziert wurden:

Situationsbeschreibung: Akteur A ist in Situation S.

Analyse der Situation: In Situationen wie S ist H die angemessene Handlung.

Rationalitätsprinzip: Akteure handeln immer situationsangemessen.

Explanandum: Daher hat Akteur A die Handlung H ausgeführt.

Das Rationalitätsprinzip gewährleistet das vernünftige Reagieren auf gegebene Bedingungen seitens der Entscheidungsträger. Obwohl das Prinzip des situationsadäquaten Handelns keineswegs immer zutreffen mag, ist es allein deshalb bei der Theoriebildung im Sinne Poppers unverzichtbar, weil die Alternativannahme der nicht am situativen Kontext ausgerichteten Handlungswahl nur vergleichsweise unklare Folgerungen erlaubt. So ist beispielsweise unklar, ob eine derartige Handlungswahl durch eine Zufallsentscheidung getroffen wird bzw. welche Entscheidungsregel denn bei nicht-zufälliger Wahl zu unterstellen ist.

Zudem gibt es ein von Myerson (1999) vorgebrachtes Argument für das Rationalitätsprinzip, das mit den Zielsetzungen sozialwissenschaftlicher Arbeiten zu tun hat. Danach erfordern zentrale gesellschaftswissenschaftliche Aufgaben jeweils Analysen von sozialen Institutionen, Organisationen und Strukturen und hierbei insbesondere die Erarbeitung und Evaluation von Reformvorschlägen. Zu klären ist für irgendwelche Verbesserungen aus der Sicht der Sozialwissenschaften, ob ein Versagen von Institutionen, Organisationen und Strukturen bei vernünftigem (und daher vorhersehbarem) Verhalten der Akteure auftreten kann.

Obwohl das Rationalitätsprinzip keine immer gültige empirische Regularität darstellt, fordert Popper (1985 [1995]) seine Voraussetzung, um den Schwerpunkt auf die Untersuchung der situativen Gegebenheiten zu legen. Nach seinen Vorstellungen soll bei regelmäßigen Abweichungen zwischen theoretischen Aussagen und empirischen Befunden das Rationalitäts- 
prinzip als fester Ausgangspunkt der handlungstheoretischen Analyse unangetastet bleiben, d.h. Theoriemodifikationen sollen sich stets auf die jeweiligen situativen Merkmale beziehen.

Das Poppersche Rationalitätsprinzip kann man auf verschiedene Arten konkretisieren. In komplexen Situationen schlägt sich Vernunft im Sinne einer Angemessenheit des Handelns anders nieder als in einfachen Situationen. Dies gilt schon aufgrund der begrenzten kognitiven Kapazitäten des Menschen (z.B. Mängel der Aufmerksamkeitslenkung, Probleme der Informationsverarbeitung), die seit geraumer Zeit bekannt sind und die oft lediglich begrenzt rationales Verhalten ermöglichen (für einen kurzen Überblick siehe Simon 1990). In kaum überschaubaren Situationen scheinen interessanterweise sehr einfache Heuristiken (wie „Take the Best“, „Recognition Heuristic“) effektiv verwendet zu werden (Gigerenzer / Todd / ABC Research Group 1999). Derartige Heuristiken lassen sich als Spielarten begrenzter Rationalität (Gigerenzer / Selten 2001) deuten, die sinnvolle Auslegungen des Popperschen Rationalitätsprinzips in komplexen Situationen sein können.

Daneben kann man das Rationalitätsprinzip auch dadurch konkretisieren, dass man Merton (1936) folgt und einfache allgemeine Annahmen trifft, die in etwa in der betrachteten Situation empirisch zutreffen (siehe hierzu und zum Folgenden Hedström 2005). In Mertons Analyse der sich selbst erfüllenden Prophezeiung besteht die Annahme beispielsweise darin, dass die gegenwärtigen Handlungen von Akteuren die Erwartungen und künftigen Handlungen anderer Individuen beeinflussen. Diese relativ unspezifische Annahme genügt in Mertons Beitrag als Spezifikation des situationsangemessenen Handelns, um die zentralen unintendierten Effekte theoretisch zu begründen.

Eine besonders wichtige Auslegung des Rationalitätsprinzips ist die „Rationalitätshypothese“, welche Rational-Choice-Soziologen (z.B. Coleman 1990; Esser 1993; Raub / Voss 1981; Wippler / Lindenberg 1987) im Allgemeinen als Mikrofundierung ihrer Erklärungen verwenden. Gemeint ist mit der Rationalitätshypothese die Annahme, dass Menschen in verschiedenen Lebensbereichen und Handlungszusammenhängen diejenigen Wege einschlagen und diejenigen verfügbaren Mittel einsetzen, die nach ihrem eigenen Urteil jeweils die Realisierung des besten Ergebnisses erlauben. Anders gesagt: Jeder Akteur versucht annahmegemäß im Rahmen von zu treffenden Entscheidungen und damit einhergehenden Handlungen, durch eine entsprechende Verwendung verfügbarer Mittel vor dem Hintergrund gegebener Vorlieben oder Präferenzen jeweils einen bestmöglichen Zustand zu erreichen. Das Postulat des optimierenden Verhaltens legt dabei keineswegs fest, dass Akteure rein egoistisch entscheiden oder nur relativ leicht beobachtbare Dinge (wie z.B. Geld) in ihrem Entscheidungskalkülen berücksichtigen; in einer soziologisch beeinflussten Erweiterung des traditionellen Menschenbildes der Ökonomik (Homo Oeconomicus) sind uneigennützige Präferenzen oder auch Vorlieben für schwer beobachtbare Dinge (wie z.B. soziale Anerkennung) prinzipiell zulässig.

Zur Begründung der Rationalitätshypothese kann man u.a. argumentieren, dass sich individuelle Abweichungen von derartigem rationalen Verhalten im Aggregat oft gegenseitig neutralisieren (z.B. Hernes 1992; Goldthorpe 1998). Weiter kann man Lernprozesse unterstellen - die Rationalitätshypothese dürfte insbesondere dann im Durchschnitt zutreffende Verhaltensvorhersagen erlauben, wenn sich relativ erfahrene Akteure in hinreichend wichtigen und transparenten Entscheidungssituationen befinden (z.B. Binmore 1998).

Dennoch ist die Voraussetzung optimierenden Verhaltens eine heroische Annahme. Sie setzt voraus, dass man seine Vorlieben, die Handlungsalternativen und ihre wahrscheinlichen Folgen sowie etwaige Beschränkungen kennt. Klarerweise handelt es sich bei der Rationalitätshypothese um eine erhebliche Zuspitzung der bereits idealisierenden Prämisse des situationsgerechten und daher vernünftigen menschlichen Handelns. Diese Eingrenzung hat 
freilich ihren Preis: Die jeweilige Konkretisierung des Optimierungsgedankens bei Erklärungen hängt üblicherweise von den Annahmen über das betrachtete Sozialsystem ab (Arrow 1990). Aufgrund der Wechselwirkung zwischen Kontext und Verhalten erscheint die Rationalitätshypothese insbesondere als geeignete Spezifikation der Popperschen Annahme des situationsangemessenen Handelns, wenn man hinreichend transparente und einfache Situationen untersucht.

Die Rationalitätshypothese wird nicht nur von einer überschaubaren Teilmenge der Soziologen, sondern weithin in der Ökonomik und der amerikanischen Politikwissenschaft verwendet. Rational-Choice-Soziologen, die sich an den Popperschen Vorschlägen zur situationsbezogenen Theoriebildung orientieren, betonen dabei nahezu immer, dass strukturelle Gegebenheiten die Verhaltenswahlen und deren Makrokonsequenzen mitprägen. Es existieren danach stets Makroeinflüsse auf das individuelle Entscheidungsverhalten (Makro-MikroÜbergang). Zudem erzeugt erst das Zusammenwirken der einzelnen Verhaltensweisen die zu erklärenden Beziehungen zwischen Makrovariablen, so dass der jeweiligen Kombinationslogik individueller Handlungen (z.B. Abstimmungsregel, Marktaggregation) wesentliche Bedeutung zukommen kann (Mikro-Makro-Übergang). Nach der Überzeugung verschiedener Autoren (z.B. Coleman 1990; Wippler / Lindenberg 1987) sollten soziologische Erklärungen somit Annahmen rationalen Handelns verwenden, ohne dass dabei strukturelle Einflüsse (wie z.B. institutionelle Regelungen, Organisationsformen oder Netzwerkbeziehungen) für individuelle Entscheidungen und kollektive Ausgänge vernachlässigt werden.

Für die Erklärung der letztlich relevanten sozialen Sachverhalte und Abläufe sind dabei normalerweise die existierenden Gleichgewichtszustände auf der Makroebene zentral, die sich durch die Kombination der individuellen Verhaltensweisen ergeben und deren Untersuchungen theoretische Folgerungen erbringen. Damit bestehen enge Parallelen zu Gary S. Beckers (z.B. 1976; 1996) Deutung der mikroökonomischen Methodologie als allgemeinen Ansatz zur Untersuchung vielfältiger sozialer Phänomene und Prozesse. Wie Sober (1983) bemerkt, spezifizieren die damit einhergehenden Gleichgewichtserklärungen allerdings üblicherweise nicht, wie ein Gleichgewichtszustand erreicht wird. Typischerweise vernachlässigen sie etwaige kausale Dynamiken und konzentrieren sich stattdessen auf statische Endpunkte oder dauerhafte Zustände. Aber selbst wenn man z.B. aufgrund dieses Mangels den Rational-Choice-Ansatz nicht akzeptiert, ergibt sich aus den bisherigen Ausführungen die Notwendigkeit erklärender Theorie und damit der Erörterung einer entsprechenden Theorieauffassung.

\section{Theoriekonzeption und Theoriebildung}

In den Sozialwissenschaften gibt es unterschiedliche Verwendungen für den Begriff „Theorie“ - oftmals wird er nicht nur im Zusammenhang mit Erklärungen, sondern auch als Synonym für z.B. Beschreibungen und Klassifikationen gebraucht. Vor relevanten Prinzipien und Anforderungen der Theoriekonstruktion ist daher die hier relevante Theorieauffassung zu konkretisieren.

\subsection{Theorie: Begriff und Komponenten}

In der Soziologie werden u.a. Vermutungen über statistische Assoziationen, mathematische Modelle, sozialphilosophische Beiträge, Reflektionen über soziale Probleme oder Zeitdiagnosen allesamt als Theorien bezeichnet. In seinem Buch über die philosophischen Grundlagen der Sozialwissenschaften stellt der Wissenschaftsphilosoph Mario Bunge (1996: 113) klar, was eine Theorie für ihn nicht ist:

„Few concepts have fared worse in the social sciences than that of theory. The worst and most popular mistakes in this regard are the following: Theory is any discourse on generalities, however obscure or 
incoherent; theory is the opposite of hard fact (a vulgar belief); theories are useless: only data and actions are valuable; theories are general orientations or approaches; theories are the same as hypotheses; theories are collections of definitions".

Aus der Perspektive Bunges vermeiden praktisch alle führenden Felder der wissenschaftlichen Forschung derartige Deutungen des Theoriebegriffes. Vielmehr beziehen sie sich in ihrer Arbeit allesamt auf weitgehend dieselbe Konzeption. Danach ist eine Theorie ein hypothetisch-deduktives System, d.h. eine Menge von Hypothesen, in der deduktive Argumentationsketten möglich sind. Anders gesagt: Eine Theorie besteht aus Behauptungen, von denen einige Annahmen darstellen und die übrigen durch logische Ableitungen aus ihnen gewonnen werden. Ist eine Theorie zudem an erfahrbaren Tatsachen und deren Erklärung interessiert, so haben die Annahmen und Folgerungen jeweils den Status von Hypothesen, die prinzipiell an empirischen Daten widerlegt werden können. Zusammenfassend kann man für die Natur- und Sozialwissenschaften daher eine Theorie als eine Menge von Aussagen bezeichnen, die durch deduktive Argumentationsketten verknüpft sind und sich zumindest teilweise empirisch überprüfen lassen.

Wie ein Blick in das Standardlehrbuch von Jonathan Turner (1991) zeigt, gibt es auch in der Soziologie Theorien im beschriebenen Sinne. Vergleicht man die Natur- und die Sozialwissenschaften, so trifft allerdings nach wie vor die folgende Aussage von Ernest Nagel (1961: 447f) zu:

„In no area of social inquiry has a body of general laws been established, comparable with outstanding theories in the natural sciences in scope or explanatory power or in capacity to yield precise and reliable predictions $[\ldots]$ It is also generally acknowledged that in the social sciences there is nothing quite like the almost complete unanimity commonly found among competent workers in the natural sciences as to what are matters of established fact, what are the reasonably satisfactory explanations (if any) for the assumed facts, and what are some of the valid procedures in sound inquiry. Disagreement on such questions undoubtedly occurs in the natural sciences as well. But it is usually found at the advancing frontiers of knowledge [...] In contrast, the social sciences often produce the impression that they are a battleground for interminably warring schools of thought, and that even subject matter which has been under intensive and prolonged study remains at the unsettled periphery of research".

Dennoch gibt es Erkenntnisse, die in sozialwissenschaftlichen Disziplinen zumindest weithin als Regelmäßigkeiten akzeptiert werden. Dies war übrigens schon vor Jahrzehnten der Fall: Berelson / Steiner (1964) haben eine grundlegende Übersichtsarbeit zu empirisch robusten Hypothesen aus der Psychologie und den Sozialwissenschaften vorgelegt; eine Zusammenstellung von systemtheoretisch orientierten, jedoch damals nur teilweise empirisch geprüften Hypothesen stammt von James Grier Miller (1978). Es existieren auch aktuellere Übersichtsarbeiten: Auf der Grundlage von vielfältigen empirischen Studien aus verschiedenen Disziplinen hat z.B. Donald Brown (1991) eine Liste von „human universals“ erstellt, die von Steven Pinker (2003) noch ergänzt wurde. Derartige empirisch fundierte RegelmäBigkeiten können zur Erklärung anderer sozialer Tatbestände und Prozesse herangezogen werden. Anders gesagt: Solche robuste Befunde und eventuell damit einhergehende Generalisierungen stellen geeignete Annahmen in Theorien der sozialwissenschaftlichen Disziplinen dar.

Aus der hier vertretenen Sicht empfiehlt sich jedenfalls ein eingeschränkterer Sprachgebrauch bezüglich des Wortes „Theorie“ als er in der Soziologie üblicherweise praktiziert wird. Danach hat eine Theorie beispielsweise nichts mit reflexiven Argumentationen zu tun. Auch stellt ein z.B. zeitdiagnostischer Beitrag aus dieser Perspektive keine Theorie dar. Diekmann (2007: 141) bietet eine Umschreibung des Theoriebegriffs, die mit den obigen Ausführungen und den Definitionen von Albert (1964: 27) und Opp (2005: 39) vereinbar ist:

Eine Theorie ist eine Menge von miteinander verknüpften Aussagen, von denen sich eine nichtleere Teilmenge auf empirisch prüfbare Zusammenhänge zwischen Variablen bezieht. 
Zur Sicherstellung empirischer Prüfbarkeit sollte eine Theorie hinreichend konkret und genau genug formuliert sein. Nach Diekmanns Deutung der strukturalistischen Theorieauffassung der analytischen Wissenschaftstheorie (Stegmüller 1980) besteht eine ausgearbeitete Theorie aus zwei Bestandteilen:

- Der „Kern“ umfasst die Grundannahmen, die sich auf empirisch zumeist unprüfbare Zusammenhänge beziehen und grundlegende Begriffe definieren.

- Die „Peripherie“ umfasst Regeln zur Messung der Variablen und die empirisch prüfbaren Hypothesen, die sich aus den Grundannahmen ableiten lassen.

Wird der Kern mathematisch formalisiert, so spricht man auch von den „Axiomen“ einer Theorie, aus denen durch logische Deduktion die empirisch prüfbaren „Theoreme“ als Teil der Peripherie der Theorie folgen. Eine solche formalisierte Theorie nennt man „Modell“; die voneinander unabhängigen und widerspruchsfreien Axiome haben sowohl notwendig als auch hinreichend für die Herleitung der Theoreme zu sein.

Klarerweise sind möglichst präzise Formulierungen von Theorien erstrebenswert. Die Erfolge naturwissenschaftlicher Erklärungen und ihrer Umsetzungen (z.B. Ingenieurwissenschaften) beruhen zu einem nicht geringen Teil auf der rigorosen Anwendung von Logik und Mathematik. Es verwundert daher nicht, dass sich Beispiele für Modelle v.a. in der Ökonomik, aber seit langem auch in der Politologie (z.B. Riker / Ordeshook 1973) und der Soziologie (z.B. Coleman 1964) finden.

Allerdings gibt es sogar im Zusammenhang mit solchen formalisierten Theorien Schwierigkeiten, die nur teilweise vermeidbar sind. Ein prinzipiell unnötiges Problem stellt z.B. der „Modell-Platonismus“ im Sinne von Albert (1980b) dar, weil tatsächlich nicht jede noch so triviale Einsicht formal herzuleiten ist. Eine unweigerlich auftretende Schwäche betrifft dagegen folgende Tatsache: Es gibt keine logische Möglichkeit zwischen konkurrierenden Modellen zu unterscheiden, welche einen empirisch gültigen Sachverhalt mithilfe unterschiedlicher Annahmen und Konzepte deduktiv begründen. Die letztlich ,adäquate“ Modellierung kann nur durch empirische Tests der abweichenden Voraussetzungen identifiziert werden. Die Prüfbarkeit solcher zentralen Annahmen von Theorien ist somit von wesentlicher Bedeutung - sind die jeweiligen Modellannahmen nämlich nicht empirisch prüfbar, so ist damit ein rigoroser Theorienvergleich praktisch ausgeschlossen. Derartige Schwächen reflektieren erkenntnistheoretische Grenzen. Sie treten auch dann auf, wenn man bestimmte Grundorientierungen für die Theoriebildung und ihre Folgerungen beachtet.

\subsection{Theoriebildung: Prinzipien und Konsequenzen}

Neben ihrer Widerspruchslosigkeit sollte eine Theorie eine möglichst hohe Genauigkeit und breite Anwendbarkeit aufweisen. Weiter ist eine gewisse Anschlussfähigkeit zu anderen theoretischen Überlegungen wünschenswert, wodurch eine spätere Verallgemeinerung der ursprünglichen Theorie nicht ausgeschlossen wird. Daneben gibt es weitere Anforderungen an eine Theorie. So sollen sich die empirisch prüfbaren Folgerungen einer Theorie im Regelfall keineswegs nur auf die Vergangenheit und Gegenwart beziehen (Albert 1980a). Gesucht sind also keine Erklärungen, die man lediglich als post hoc Plausibilisierungen bisheriger historischer Entwicklungen betrachten kann. Vielmehr sollen die empirisch testbaren Hypothesen auch zukünftige Zusammenhänge betreffen - idealerweise haben die von Theorien angebotenen Erklärungen jeweils Vorhersagen zu erlauben, die an der Realität scheitern können.

Nahezu immer handelt es sich bei den empirisch prüfbaren Aussagen einer Theorie um „ceteris paribus“-Hypothesen. Oftmals wird in einer Theorie ein kausaler Zusammenhang zwischen Variablen behauptet, der unter der zumeist impliziten theoretischen Prämisse gilt, dass andere Einflussfaktoren unverändert bleiben. Wünschenswert ist daher die Explikation aller anderen Variablen, die als potenziell einflussreich betrachtet werden und deren Kon- 
stanz für eine etwaige Gültigkeit der Hypothesen der Theorie vorausgesetzt wird. Man sollte also bei der Präsentation der Theorie darauf achten, dass sämtliche Einflüsse angegeben werden und die als konstant angenommenen Größen jeweils spezifiziert sind. Durch eine „ceteris paribus"-Klausel wird die Aufmerksamkeit jeweils auf eine einzelne Fragestellung gerichtet, und von weiteren, eventuell simultan ablaufenden Phänomenen und Prozessen typischerweise abstrahiert. Trotz aller berechtigten Kritik an dieser Vorgehensweise gibt es bei der Theoriebildung hierzu normalerweise keine überzeugende Alternative.

Zudem werden oftmals nur wenige Variablen bei der Theoriebildung berücksichtigt. Unabhängig von der betrachteten Erfahrungswissenschaft wird bei der Theoriebildung nämlich zumeist ein Ökonomie-Prinzip eingehalten, das auf den spätscholastischen Philosophen Ockham zurückgeht:

Alle zur Erklärung eines Sachverhaltes oder Ablaufs nicht notwendigen Voraussetzungen und Begründungen sind überflüssig (Ockhams Rasiermesser: „Eine Vielheit ist ohne Notwendigkeit nicht zu setzen“).

Unnötige Hypothesen sind demnach bei der Theoriebildung zu vermeiden. Damit werden sparsame Theorien aus methodologischer Sicht präferiert, wodurch vermeintlich ,unterkomplexe“ Erklärungen generell kein Problem darstellen. Bei Beachtung des Ökonomie-Prinzips liefert eine Theorie eine Minimalerklärung, die keineswegs inadäquat zu sein braucht - ein beobachteter Sachverhalt oder Ablauf kann durch die spezifizierten wenigen Bedingungen durchaus logisch konsistent und hinreichend erschöpfend begründet werden. Dies bedeutet allerdings nicht, dass alternative Erklärungen für die Beobachtungen ausgeschlossen sind.

Bei Befolgung des in den meisten Erfahrungswissenschaften akzeptierten Ökonomie-Prinzips der Theoriebildung ergibt sich unweigerlich die folgende Einsicht:

Jede Theorie beruht auf Prämissen, die tatsächliche Sachverhalte und Abläufe ausblenden oder nicht völlig zutreffen.

Eine Theorie stellt also immer eine gewisse Verzerrung der Wirklichkeit dar. Sie beruht auf Vereinfachungen, die Ausschnitte der Realität zweckorientiert herausheben oder vernachlässigen. Vor diesem Hintergrund erscheint eine ideale Theorie wie ein Stadtplan - sie stellt eine hilfreiche Abstraktion zum Verständnis der Realität dar. Durch die Vernachlässigung untergeordneter Aspekte und die damit verbundene Überbetonung der für die Problemstellung relevanten Zusammenhänge ist eine ideale Theorie notwendigerweise eine verzerrende Abbildung, die gleichzeitig aber höchst praktisch ist.

Eine damit verknüpfte weitere Konsequenz des Ökonomie-Prinzips der Theoriebildung besteht darin, dass eine Theorie niemals alles erklären kann. Eine Theorie besitzt lediglich Relevanz für einen begrenzten Ausschnitt der Wirklichkeit - im Idealfall werden die theoretischen Annahmen so expliziert, dass der Geltungsbereich der jeweiligen Theorie deutlich wird. Keine Theorie kann Phänomene und Prozesse erklären, die mit den von ihr ausgeblendeten Aspekten zu tun haben.

Dies gilt auch dann, wenn mit dem Objektivitätspostulat ein weiteres Prinzip der Theoriebildung beachtet wird. Entsprechend der Ausführungen von Vollmer (1975: 31f) bedeutet objektiv hier wirklichkeitsbezogen. Theoretische Aussagen beziehen sich demnach auf die als existent angenommene Realität. Sie reflektieren jedoch nicht den subjektiven Zustand irgendwelcher Personen (wie Beobachter und Theoretiker). Objektivität von theoretischen Ausführungen erfordert nach Vollmer zumindest die Erfüllung der folgenden Kriterien:

Intersubjektive Verständlichkeit: Die Aussagen müssen kommunizierbar und verstehbar sein. 
Unabhängigkeit vom Bezugssystem: Die Aussagen haben von den Perspektiven irgendwelcher Personen unabhängig zu sein.

Intersubjektive Nachprüfbarkeit: Jeder soll die Aussagen durch geeignete Methoden überprüfen können.

Unabhängigkeit von der Methodik: Folgerungen zur Korrektheit von Aussagen dürfen nicht von der Methode abhängen, die zu ihrer Prüfung verwendet wird.

Unabhängigkeit von Willkürlichkeit: Folgerungen zur Korrektheit von Aussagen dürfen nicht auf willkürlichen Beschlüssen oder Konventionen beruhen.

Eine unmittelbare Konsequenz des Objektivitätspostulats ist die Ablehnung subjektiv geprägter Interpretationen im Zuge der qualitativ orientierten Sozialforschung und ihre induktive Weiterverarbeitung in logisch fragwürdigen Verallgemeinerungen und Theoriekonstruktionen (siehe Holweg (2005) für weitere methodologische Kritikpunkte). Ebenso sind an spezifische Theorieentwürfe angepasste Methoden unzulässig, falls dadurch die Objektivität des Vorgehens in Frage gestellt wird. Wohl gemerkt wird Theoriebildung im Gefolge qualitativer Sozialforschung durch das Objektivitätspostulat keineswegs ausgeschlossen. Verlangt wird allerdings, dass man sich unabhängig vom relevanten Datenmaterial um Objektivität und damit um die Erfüllung der genannten Kriterien bemüht. Zur Verdeutlichung ist eine Illustration sinnvoll.

\subsection{Beispiel für eine Theorie}

Erklärt werden soll die langfristige Beziehung zwischen Wohlstand und Gewalt in modernen westlich geprägten Gesellschaften. Dabei wird auf eine handlungstheoretische Fundierung verzichtet, um die erwähnte Unabhängigkeit des Theoriekonzepts vom methodologischen Individualismus und dem Rational-Choice-Ansatz zu belegen. Stattdessen werden demographische Entwicklungen mit sozialwissenschaftlichen Einsichten zu Ausbildungsinvestitionen und Interaktionshäufigkeiten kombiniert. Dadurch resultiert eine Theorie der Gewaltrate, die mit der z.B. von Heinsohn (2003) vertretenen These einer erhöhten Kriegsgefahr bei einem hohen Anteil junger Männer vereinbar scheint und daher eine gewisse Anschlussfähigkeit besitzt. Konkret postuliert werden folgende Annahmen:

A1: In westlich geprägten Gesellschaften mit geringer $\mathrm{Zu}$ - und Abwanderung geht mit gestiegenem Wohlstand (d.h. Erhöhung des Pro-Kopf-Einkommens bei weitgehend konstanten Preisen) eine Verminderung der durchschnittlichen Kinderzahl pro gebärfähiger Frau einher.

A2: Bei zunehmendem Einkommen und sonst weitgehend konstanten Preisen geben Eltern in westlich geprägten Gesellschaften mehr für die Qualifizierung jedes Kindes aus.

A3: Ungefähr gleichaltrige Personen haben im Durchschnitt eher soziale Interaktionen miteinander als Personen mit höherem Altersunterschied.

A4: Die Zahl der Gewalthandlungen steigt mit der relativen Häufigkeit von Interaktionen zwischen jungen Männern (15-30 Jahre), sinkt aber mit den im Durchschnitt absolvierten Schul- und Ausbildungsjahren.

Die ersten beiden Zusammenhangsaussagen finden sich in der Literatur über Fertilität (z.B. Becker 1960; Becker / Lewis 1973). Wolf (1996) informiert über die drittgenannte These zur Homophilie und deren vielfältige empirische Stützung durch Befunde zu Ähnlichkeiten bei Alter, beruflicher Stellung, Einstellungen, Familienstand, Geschlecht und Konfession. Die viertgenannte Hypothese ist ein Standardbefund der Kriminologie (z.B. Hirschi / Gottfredson 1994). 
Die ersten drei Annahmen verweisen auf kausale Mechanismen der Theorie, die miteinander verknüpft sind: Fertilitätsreduktion durch Wohlstandsmehrung, Wettbewerbsintensivierung innerhalb der jungen Generation und daher verstärkte elterliche Qualifizierungsinvestitionen sowie Häufigkeitsreduktion der Interaktionen zwischen Jungen im Zuge der Homophilie-Gesetzmäßigkeit. Zieht man überdies die letztgenannte Aussage heran, so führt ihre Kombination zu drei Folgerungen:

F1: In westlich geprägten Gesellschaften mit geringer Zu- und Abwanderung nimmt die Zahl junger Männer bei wachsendem Wohlstand ab, wodurch sich auch die relative Häufigkeit ihrer Interaktionen vermindert.

F2: Mit erhöhten Einkommen verbessert sich von Generation zu Generation das durchschnittliche Bildungsniveau in westlich geprägten Gesellschaften mit geringer $\mathrm{Zu}$ - und $\mathrm{Ab}$ wanderung.

F3: Die Rate der Gewalthandlungen (d.h. ihr Anteil an sämtlichen bekannten Delikten im fraglichen Zeitraum) fällt in westlich geprägten Gesellschaften bei geringer $\mathrm{Zu}$ - und Abwanderung mit wachsendem Wohlstand, falls die Zahl der bekannten Delikte nicht stärker sinkt als die Zahl der Gewalttaten.

Die vorläufige Akzeptanz oder Ablehnung sämtlicher Hypothesen ist jeweils eine empirische Frage. In diesem Beispiel gibt es keine Aussage, die nicht einer empirischen Prüfung unterzogen werden kann. Allerdings sind hierfür einige Zusatzannahmen zu treffen: So ist beispielsweise festzulegen, welcher Zeithorizont für eine etwaige Gültigkeit der Aussagen unterstellt werden kann. Weiter ist zu bestimmen, was eine westlich geprägte Gesellschaft ist (Handelt es sich um Nationalstaaten? Wenn ja, gehören Japan oder die früheren Ostblockstaaten dazu?). Zudem ist zu bestimmen, was eine geringe $\mathrm{Zu}$ - und Abwanderung ist und ob nur polizeilich erfasste Delikte gemeint sind.

Klar gestellt sind in der Theorie dagegen die jeweils konstant zu haltenden Variablen, d.h. es wird auf unspezifizierte „ceteris paribus“-Klauseln verzichtet. Überdies werden sämtliche Konzepte definiert. Etwaige Versuche einer empirischen Prüfung der genannten Annahmen und Folgerungen können daher vor dem Hintergrund dieser Operationalisierungsvorschläge erfolgen. Damit wird der Forderung nach Objektivität für die relevanten Hypothesen und deren Prüfung Rechnung getragen.

Allerdings ist die gewählte Illustration sehr einfach gehalten, weil auf eine Vielzahl weiterer möglicher Faktoren überhaupt nicht eingegangen wird. Man hätte ja z.B. thematisieren können, dass Gewalttätigkeit biologische Ursachen (Aggression als Folge von Testosteron) hat. Auch hätte man darauf verweisen können, dass Gewalttätigkeit nicht selten Erfahrungen in der Sozialisation (wie etwa gewalttätige Eltern oder Geschwister) reflektiert, durch die Eingebundenheit in bestimmte Gruppen (z.B. Jugendbanden) und die Konformität mit deren Normen bedingt ist oder Probleme mit gewissen psychoaktiven Substanzen (wie Alkohol, Amphetamine oder Kokain) widerspiegelt. Weiter hätte man argumentieren können, dass Gewalthandlungen auf bestimmte Veränderungen (u.a. Einführung des Privatfernsehens und damit erhöhter kindlicher Fernsehkonsum, Verbreitung von Kampfsportarten und verstärkter Körperkult, Verfügbarkeit von billiger leistungsfähiger Hardware und gewaltorientierten Computerspielen) zeitverzögert reagiert. Zudem hätte man herausarbeiten können, dass Gewalttätigkeit häufig durch Personen mit Migrationshintergrund erfolgt, oftmals mit Arbeitslosigkeit zusammenhängt und insbesondere in größeren Städten zu beobachten ist. Die Ausblendung dieser möglichen Einflüsse und Korrelate reflektiert im Übrigen keine „Unterkomplexität“ der Erklärung, sondern das Ökonomieprinzip der Theoriebildung, wonach unnötige Hypothesen vermieden werden sollen. Zu fragen ist jedoch nach der Wirklichkeitsnähe einer derart reduzierten Theorie und ihrer Voraussetzungen. 


\section{Realitätsnähe von Theorieannahmen}

In wissenschaftsphilosophischen Übersichtsarbeiten (z.B. Godfrey-Smith 2003; Rosenberg 2006) werden Realismus und Instrumentalismus als Positionen zur Wirklichkeitsnähe von theoretischen Postulaten kontrastiert. Der Realismus geht bekanntlich von der Existenz einer Wirklichkeit aus, die nicht nur eine Vorstellung ist und deren wahre Strukturen annähernd verstehbar sind. Im Idealfall weisen Theorien aus dieser Sicht jeweils eine weitgehende Korrespondenz mit der Wahrheit auf. Theoretische Annahmen sollten daher hinreichend realistisch und nicht empirisch falsch sein.

Der Instrumentalismus ist dagegen eine Spielart des Pragmatismus. Er betont nicht die Korrektheit von Theorien, sondern allein deren Nützlichkeit. Theorien werden nicht geglaubt oder bezweifelt. Sie sind vielmehr Instrumente für die Begründung von testbaren Aussagen. Dabei kommt es nicht auf den deskriptiven Gehalt der getroffenen Voraussetzungen an theoretische Annahmen sind weitgehend beliebig wählbar. Wesentlich ist nur die empirische Bewährung der auf ihrer Grundlage hergeleiteten Hypothesen. Dadurch wird reflektiert, dass jede Theorie unweigerlich von mehr oder weniger vielen Aspekten abstrahiert und daher ohnehin kein getreues Abbild der Wirklichkeit darstellt.

Instrumentalistische und realistische Perspektiven finden sich in methodologischen Diskussionen der Sozialwissenschaften. Auch dort wird seit langem gefragt, inwieweit Voraussetzungen von Theorien möglichst realitätsgerecht sein sollten (z.B. Albert 1964; Blaug 1980; Brinkmann 1997; Manicas 2006; Opp 2005). In einem bekannten methodologischen Beitrag argumentierte insbesondere der Ökonom Milton Friedman (1953), dass der deskriptive Gehalt der Prämissen bei der Theoriebildung unwesentlich sei. Für Friedman stellt eine Theorie ein Vorhersageinstrument dar, dessen Güte nur an der empirischen Bewährung ihrer Folgerungen (also ihrem Prognoseerfolg) gemessen werden kann. In der Soziologie nimmt u.a. Jasso (1988) eine instrumentalistische Position ein, die sich an Friedman orientiert.

Gegen Friedmans Argumentation kann man mit Herbert Simon (1963) darauf verweisen, dass Theorien normalerweise Erklärungen liefern. Daher ist die gesamte logische Erklärungskette bei der Beurteilung und Überprüfung einer Theorie zu berücksichtigen. Eine theoretisch begründete Vorhersage kann man vor diesem Hintergrund lediglich als eine Probe für die Korrektheit einer Erklärung ansehen, aber keinesfalls als Endzweck der Theoriebildung. Empirisch falsche Annahmen sind schon deshalb möglichst zu vermeiden, weil sie nicht korrekt beantworten können, warum etwas passiert.

Dies gilt auch für unrealistische Annahmen, die zum Zwecke der mathematischen Vereinfachung getroffen werden - das Interesse an eleganten Theorien kann sie nicht rechtfertigen, da Eleganz keinen Wahrheitsbezug mit sich bringt. Aussagen über erfundene Welten haben nichts mit der Wirklichkeit zu tun und erklären diese daher auch nicht. Hedström (2005), der eine realistische Position einnimmt, wirft insbesondere einigen Rational-Choice-Theoretikern ihren Instrumentalismus vor, weil sie nach seiner Ansicht wirklichkeitsferne Annahmen zum Zwecke der Modellierung allzu leichtfertig einführen.

Dabei übersieht er jedoch eine wesentliche Forderung des Spieltheoretikers Robert Aumann (1985): Sozialwissenschaftliche Theorien sollten weniger nach der Realitätsnähe und Plausibilität ihrer Annahmen bewertet werden, sondern eher nach der Zahl und Reichweite ihrer Folgerungen für das Verständnis der Wirklichkeit. Aus dieser Sicht sind sozialwissenschaftliche Theorien daran zu bemessen, was sie an prinzipiell widerlegbaren Hypothesen in der Ökonomik, Politologie und Soziologie schon generiert haben und derzeit noch anregen. Impliziert eine Theorie auf der Grundlage unrealistischer Annahmen vielfältige empirisch prüfbare Zusammenhänge, so erscheint sie für das Verständnis der Welt fruchtbarer als eine Theorie mit zwar realitätsnäheren Annahmen, aber weniger relevanten Implikationen. Bei- 
spielsweise ist die Annahme begrenzt rational handelnder Akteure im Sinne von Herbert Simon (1990) sicher deskriptiv korrekter als die Annahme vollständig rational handelnder Akteure; allerdings ist die Rationalitätshypothese bisher die weitaus fruchtbarere Prämisse bei der Herleitung empirisch prüfbarer Aussagen gewesen.

Theoriebildung ist daher nicht nur ein fortlaufender Prozess der Prüfung und Überarbeitung anhand der empirischen Evidenz zu den jeweils testbaren Theoriefolgerungen. Vielmehr ergibt sich bei Akzeptanz der Aumannschen Gedanken noch eine weitere wichtige Einsicht: Theoriebildung hat immer auch mit der Festlegung von Annahmenkombinationen zu tun, die sich als besonders fruchtbar für die Genese von empirisch relevanten Aussagen erwiesen haben und erweisen. Unrealistische Annahmen erscheinen keineswegs als überflüssig, wenn sie in diesem Sinne das Verständnis der Realität erweitern.

Zudem bilden Theorien die Wirklichkeit niemals wahrheitsgetreu ab, weil sie stets auf bewussten Vereinfachungen z.B. aufgrund des Ökonomie-Prinzips beruhen. Eine gewisse Verzerrung der Realität ist im Rahmen der Theoriebildung mithin unvermeidbar. Aus der Sicht der quantitativ orientierten Sozialforschung gibt es daneben ein von Esser (1993) angeführtes Argument zugunsten des Instrumentalismus: Empirische Prüfungen von Theorien beruhen stets auf bestimmten Annahmen, die im Test keineswegs empirisch untersucht werden können.

Insgesamt werden praktisch immer unprüfbare und verzerrende Annahmen vorliegen, wenn man empirisch testbare Theorien betrachtet. Empirische Untersuchungen erlauben jedoch vorläufige Einsichten über etwaige Vereinfachungen und ihre Angemessenheit. Lassen sich die prüfbaren Aussagen einer Theorie bei regelmäßigen empirischen Tests nämlich nicht widerlegen, so sind die in den Theorieannahmen vernachlässigten Aspekte offenbar tatsächlich von nachrangiger Wichtigkeit. Es wurden dann wahrscheinlich ,adäquate“ Annahmen getroffen, d.h. es wurde bei der Theoriebildung von vermutlich unwesentlichen Fakten abstrahiert. Auch weil empirische Bewährungen dies aber keineswegs mit Sicherheit nachweisen können, ist theoretisches Wissen im Allgemeinen nur vorläufig.

Ergeben sich Abweichungen zwischen empirischen Befunden und den theoretischen Hypothesen in systematischen Studien, so sind durch die bisherigen Theorieannahmen vernachlässigte Aspekte vermutlich von stärkerer Bedeutung als ursprünglich gedacht, was bei Modifikationen der jeweiligen Theorie zu berücksichtigen ist. Man kann die Theoriebildung daher auch als Vorgang beschreiben, dessen Prinzip in einem bewussten Lernen aus Fehlern und der laufenden Vornahme von Korrekturen besteht. Voraussetzung ist dabei die dauernde Konfrontation von empirischen prüfbaren Hypothesen mit Daten und die fortwährende Bereitschaft zur Revision theoretischer Überlegungen aufgrund von empirischen Gegebenheiten.

Dabei bezweifelt niemand die Notwendigkeit von Abstraktionen bei der Theoriebildung. Allerdings müssen sich Annahmen für den jeweiligen Zweck der Theoriebildung eignen. Daneben ist zu beachten, was Alan Musgrave (1981) in einem Beitrag zu Friedmans These der Irrelevanz theoretischer Postulate betont: Nicht alle Annahmen spielen dieselbe Rolle in einer Theorie, weshalb Friedmans Pauschalurteil keinesfalls für alle unterscheidbaren Typen von Voraussetzungen gilt. Die unvermeidlichen Vereinfachungen einer Theorie sind im Idealfall daher so vorzunehmen, dass die damit einhergehenden Folgerungen möglichst nicht von einzelnen speziellen Annahmen abhängen. Sind solche Postulate aber unumgänglich, so sollten diese wesentlichen Annahmen möglichst empirisch prüfbar und nicht völlig kontrafaktisch sein.

Dies erscheint u.a. deshalb sinnvoll, weil es häufig konkurrierende Theorien gibt, die auf unterschiedlichen Annahmen beruhen, aber zu ähnlichen Folgerungen gelangen. Um in ei- 
nem empirischen Anwendungsfall zwischen solchen Theorien unterscheiden zu können, ist die Prüfung zentraler Annahmen erforderlich. Hintergrund dieser Aussage ist die logische Tatsache, dass aus falschen Prämissen wahre Konklusionen folgen können, aber aus wahren Konklusionen nicht auf die Wahrheit der Prämissen geschlossen werden kann. Somit gibt es keine logische Möglichkeit zwischen konkurrierenden Theorien zu diskriminieren, welche einen empirisch gültigen Sachverhalt mithilfe unterschiedlicher Annahmen deduktiv begründen - die letztlich angemessene Theorie kann nur durch empirische Befunde zu den abweichenden Voraussetzungen identifiziert werden.

Theorienvergleiche sind jedoch keineswegs nur empirischer Natur. $\mathrm{Zu}$ fragen ist daher nach Kriterien des Vergleichs konkurrierender Theorien und Aspekten der Wissensentwicklung.

\section{Theorienkonkurrenz und Erkenntnisfortschritt}

Für einen Theorienvergleich lassen sich verschiedene Gütekriterien spezifizieren: Eine Theorie erscheint danach umso besser, je mehr Phänomene und / oder Prozesse sie erklärt und voraussagt, je präziser sie ist, je weniger spezifische Begriffe und unprüfbare Aussagen sie enthält, je größer die Beobachtbarkeit und / oder Operationalisierbarkeit ihrer Konstrukte ist und je öfter ihre prüfbaren Folgerungen durch empirische Befunde nicht widerlegt werden konnten. Aufgrund derartiger Kriterien kann man rivalisierende Theorien als mehr oder weniger nützlich für das Verständnis der Welt kategorisieren und sich im Idealfall für eine bestimmte Theorie entscheiden.

Gerade weil aber auch die derzeit beste Theorie jeweils nur vorläufiges Wissen darstellt, gibt es Erkenntnisfortschritt. Theorien können durch andere hypothetisch-deduktive Systeme ersetzt werden, sofern letztere z.B. eine durchgehend bessere empirische Passung als erstere haben. Auch wenn man die Theoriegeladenheit von empirischen Untersuchungen berücksichtigt, können sich die Aussagen von Theorien aufgrund von entsprechenden Beobachtungen als mit der Wirklichkeit korrespondierend erweisen (z.B. kugelförmige Gestalt der Erde), während Aussagen alternativer Theorien keine derartige Korrespondenz haben (z.B. scheibenförmige Gestalt der Erde). Diese Möglichkeit reflektiert Poppers (z.B. 1934 [1994]) Auffassung, wonach mit der Falsifikation theoretischer Aussagen im Rahmen von empirischen Untersuchungen eine fortgesetzte Sequenz von Versuch und Irrtum einhergeht, die zu einer Theoriendynamik führt. Aus dieser Perspektive ergibt sich Erkenntnisfortschritt, sofern eine stärkere Annäherung an die tatsächlichen Gegebenheiten durch Theoriemodifikationen möglich wird.

Allerdings erfolgt die Entwicklung von Theorien und damit der Fortschritt in der Wissenschaft keineswegs kontinuierlich. Wie Kuhn (1962) in seiner Arbeit über die Forschungsdynamik betont, existiert normalerweise in einer wissenschaftlichen Disziplin ein Paradigma, d.h. eine weithin akzeptierte und nicht weiter hinterfragte Grundansicht, die den Wissenschaftlern eine Orientierungshilfe zur Einordnung und Bearbeitung der interessierenden Sachverhalte und Abläufe bietet. Vor dem Hintergrund des Paradigmas einer Wissenschaft ergeben sich Fragen und Methoden zu deren Beantwortung. In der Praxis werden daher prinzipiell falsifizierte Hypothesen keineswegs zwingend verworfen; sie werden üblicherweise unter Beibehaltung der Grundansicht so verändert, dass Widersprüche möglichst vermieden werden können. Erst wenn Diskrepanzen zwischen Empirie und Theorie hinreichend häufig auftreten, werden diese als Anomalien betrachtet, die das Paradigma untergraben und letztlich zu einer Grundlagenkrise und Umorientierung der jeweiligen Wissenschaft (Paradigmenwechsel) führen.

Poppers und Kuhns Ideen sind von Lakatos (1974) aufgegriffen und weiterentwickelt worden. Danach geht es in der Wissenschaft um die Konkurrenz von Forschungsprogrammen. 
Ein Forschungsprogramm verweist auf ein Bündel von Theorien, die jeweils auf nicht falsifizierbaren Grundgedanken beruhen, aber mit empirisch widerlegbaren Hypothesen einhergehen. Nach der Auffassung von Lakatos besteht typischerweise ein Wettbewerb von solchen Programmen, der letztlich durch empirische Untersuchungen entschieden wird.

Betrachtet man die Soziologie, so kann man freilich kaum von einem vorherrschenden Paradigma im Sinne von Kuhn oder einem tatsächlichen Wettstreit von Forschungsprogrammen im Sinne von Lakatos sprechen - im Fach existieren überwiegend unvereinbare Theorieansätze, die zudem keine hinreichend engen Substitute füreinander darstellen. Dies reflektiert vermutlich u.a., dass es aufgrund fehlender empirischer Evidenz unmöglich und unzweckmäßig sein kann, sich für lediglich einen Theorieansatz zu entscheiden.

Wie erwähnt erschwert die Inkompatibilität der theoretischen Ansätze in der Soziologie und ihre fehlende Konkurrenz den Aufbau eines weithin akzeptierten Wissensbestandes. Vermutlich deshalb erscheint der Erkenntnisfortschritt im Fach oft unklar, obwohl sich theoretische Vorstellungen in der Soziologie zweifellos verändern (z.B. Auf- und Abstieg des Strukturfunktionalismus) und sich Methoden der empirischen Sozialforschung schon aufgrund des technischen Fortschritts weiterentwickeln (z.B. PC-gestuitzte Experimente, Varianten der Online-Befragung). Wenn von einer Wissensentwicklung in der Soziologie gesprochen wird, dann bezieht sich diese Aussage eigentlich fast immer auf den Zuwachs an Aufsätzen und Büchern in einem bestimmten Teilgebiet des Faches vor dem Hintergrund eines bestimmten theoretischen Ansatzes und der damit verknüpften methodischen Vorgehensweisen. Nicht gemeint sind dagegen empirisch bestätigte und theoretisch fundierte kausale Zusammenhänge über das soziale Handeln, soziale Ordnungen und den sozialen Wandel.

Dies ist schon alleine deshalb bemerkenswert, weil sich die Soziologie ja seit jeher mit ihren Möglichkeiten und Grenzen beschäftigt. So wird seit langem betont, dass die Soziologie auch sich selbst erforscht, also eine reflexive Disziplin ist. Leider hat diese Reflexion bislang nicht dazu geführt, dass die Theoriebildung im Fach mehrheitlich hinterfragt wird oder dass sie sich gar an erfolgreicheren Vorbildern orientiert. Wenn die Sozialtheorie auch deshalb kaum noch gesellschaftliche Auswirkungen hat, dann stellt sich nach Coleman (1990) die Frage nach der Daseinsberechtigung der Soziologie. Die Übernahme des diskutierten Theorieverständnisses würde vermutlich langfristig helfen, die soziologische Theorie zu rehabilitieren.

\section{Literatur}

Albert, Hans (Hrsg.) (1964): Theorie und Realität. Ausgewählte Aufsätze zur Wissenschaftslehre der Sozialwissenschaften, Tübingen.

Albert, Hans (1980a): Theorie und Prognose in den Sozialwissenschaften, in: Ernst Topitsch (Hrsg.), Logik der Sozialwissenschaften, 10. Auflage, Königstein, S. 126-143.

Albert, Hans (1980b): Modell-Platonismus. Der neoklassische Stil des ökonomischen Denkens in kritischer Beleuchtung, in: Ernst Topitsch (Hrsg.), Logik der Sozialwissenschaften, 10. Auflage, Königstein, S. 406-434.

Arrow, Kenneth J. (1990): Economic Theory and the Hypothesis of Rationality, in: John Eatwell / Murray Milgate / Peter Newman (Hrsg.), The New Palgrave: Utility and Probability, New York, S. 25-37.

Aumann, Robert J. (1985): What is Game Theory Trying to Accomplish?, in: Robert J. Aumann, Collected Papers, Vol. I, Cambridge, S. 5-46. 
Becker, Gary S. (1960): An Economic Analysis of Fertility, in: Ansley J. Coale (Hrsg.), National Bureau of Economic Research: Demographic and Economic Change in Developed Countries, Princeton, S. 209-231.

Becker, Gary S. (1976): The Economic Aproach to Human Behavior, Chicago.

Becker, Gary S. (1996): Accounting for Tastes, Cambridge.

Becker, Gary S. / H. Gregg Lewis (1973): On the Interaction between Quantity and Quality of Children, in: Journal of Political Economy 81, S. 279-288.

Berelson, Bernard / Gary A. Steiner (1964): Human Behavior. An Inventory of Scientific Findings, New York - Chicago - Burlingame.

Binmore, Ken (1998): Game Theory and the Social Contract. Volume 2: Just Playing, Cambridge.

Blau, Peter M. (1964): Exchange and Power in Social Life, New York.

Blaug, Mark (1980): The Methodology of Economics, Cambridge.

Boudon, Raymond (1980): Die Logik gesellschaftlichen Handelns, Darmstadt.

Boudon, Raymond (1987): The Individualistic Tradition in Sociology, in: Jeffrey C. Alexander / Bernhard Giesen / Richard Münch / Neil J. Smelser (Hrsg.), The Micro-Macro Link, Berkeley, S. 47-70.

Brinkmann, Gerhard (1997): Analytische Wissenschaftstheorie, 3. überarbeitete und erweiterte Auflage, München.

Brodbeck, May (Hrsg.) (1969): Readings in the Philosophy of the Social Sciences, New York.

Brown, Donald E. (1991): Human Universals, New York.

Bunge, Mario (1996): Finding Philosophy in Social Science, New Haven - London.

Calhoun, Craig J. (1996): What Passes for Theory in Sociology?, in: Sociological Theory 14, S. 1-2.

Coleman, James S. (1964): Introduction to Mathematical Sociology, New York.

Coleman, James S. (1973): The Mathematics of Collective Action, London.

Coleman, James S. (1990): Foundations of Social Theory, Cambridge.

Diekmann, Andreas (2007): Empirische Sozialforschung, 18. vollständig überarbeitete und erweiterte Neuausgabe, Reinbek.

Diekmann, Andreas / Thomas Voss (Hrsg.) (2004): Rational-Choice-Theorie in den Sozialwissenschaften. Anwendungen und Probleme, München.

Emerson, Richard M. (1962): Power-Dependence Relations, in: American Sociological Review 27, S. 31 41.

Esser, Hartmut (1993): Soziologie: Allgemeine Grundlagen, Frankfurt / Main.

Friedman, Milton (1953): The Methodology of Positive Economics, in: Milton Friedman, Essays in Positive Economics, Chicago, S. 3-43.

Gigerenzer, Gerd / Reinhard Selten (Hrsg.) (2001): Bounded Rationality. The Adaptive Toolbox, Cambridge.

Gigerenzer, Gerd / Peter Todd / ABC Research Group (1999): Simple Heuristics that Make Us Smart, Oxford.

Godfrey-Smith, Peter (2003): Theory and Reality. An Introduction to the Philosophy of Science, Chicago - London.

Goldthorpe, John (1998): Rational Action Theory for Sociology, in: British Journal of Sociology 49, S. 167-192.

Hedström, Peter (2005): Dissecting the Social. On the Principles of Analytical Sociology, Cambridge.

Hedström, Peter / Richard Swedberg (Hrsg.) (1998): Social Mechanisms. An Analytical Approach to Social Theory, Cambridge.

Heinsohn, Gunnar (2003): Söhne und Weltmacht. Terror im Aufstieg und Fall der Nationen, Zürich.

Hempel, Carl G. (1965): Aspects of Scientific Explanation and Other Essays in the Philosophy of Science, New York. 
Hempel, Carl G. / Paul Oppenheim (1948): Studies in the Logic of Explanation, in: Philosophy of Science 15, S. 135-175.

Hernes, Gudmund (1992): We are Smarter Than We Think: A Rejoinder to Smelser, in: Rationality and Society 4, S. 421-436.

Hirschi, Travis / Michael R. Gottfredson (Hrsg.) (1994): The Generality of Deviance, New Brunswick.

Holweg, Heiko (2005): Methodologie der qualitativen Sozialforschung. Eine Kritik, Bern.

Homans, George C. (1974): Social Behavior. Its Elementary Forms, 2. erweiterte Auflage, New York.

Jasso, Guillermina (1988): Principles of Theoretical Analysis, in: Sociological Theory 6, S. 1-20.

Joas, Hans / Wolfgang Knöbl (2004): Sozialtheorie. Zwanzig einführende Vorlesungen, Frankfurt / Main.

Koertge, Noretta (1975): Popper's Metaphysical Research Program for the Human Sciences, in: Inquiry 18 , S. 437-462.

Kuhn, Thomas S. (1962): The Structure of Scientific Revolutions, Chicago.

Lakatos, Imre (1974): Falsifikation und die Methodologie wissenschaftlicher Forschungsprogramme, in: Imre Lakatos / Alan Musgrave (Hrsg.), Kritik und Erkenntnisfortschritt, Braunschweig, S. 89-189.

Lindenberg, Siegwart (1996): Constitutionalism and Relationalism: Two Versions of Rational Choice, in: Jon Clark (Hrsg.), James S. Coleman, London, S. 299-311.

Manicas, Peter T. (2006): A Realist Philosophy of Social Science. Explanation and Understanding, Cambridge.

Mayntz, Renate (2004): Mechanisms in the Analysis of Social Macro-Phenomena, in: Philosophy of the Social Sciences 34, S. 237-259.

Merton, Robert K. (1936): The Unanticipated Consequences of Purposive Social Action, in: American Sociological Review 1, S. 894-904.

Merton, Robert K. (1957): Social Theory and Social Structure, New York.

Mikl-Horke, Gertraude (2001): Soziologie. Historischer Kontext und Theorie-Entwürfe, 5. Auflage, München - Wien.

Miller, James Grier (1978): Living Systems, New York.

Morgan, Stephen L. / Christopher Winship (2007): Counterfactuals and Causal Inference. Methods and Principles for Social Research, Cambridge.

Münch, Richard (2002): Soziologische Theorie, Band 1: Grundlegung durch die Klassiker, Frankfurt / Main.

Münch, Richard (2003): Soziologische Theorie, Band 2: Handlungstheorie, Frankfurt / Main.

Münch, Richard (2004): Soziologische Theorie, Band 3: Gesellschaftstheorie, Frankfurt / Main.

Musgrave, Alan (1981): „Unreal Assumptions“ in Economic Theory: The F-Twist Untwined, in: Kyklos 34, S. 377-387.

Myerson, Roger B. (1999): Nash Equilibrium and the History of Economic Theory, in: Journal of Economic Literature 37, S. 1067-1082.

Nagel, Ernest (1961): The Structure of Science, New York -Chicago - Burlingame.

Opp, Karl-Dieter (2005): Methodologie der Sozialwissenschaften, 6. Auflage, Wiesbaden.

Pearl, Judea (2000): Causality. Models, Reasoning, and Inference, Cambridge.

Pinker, Steven (2003): Das unbeschriebene Blatt: Die moderne Leugnung der menschlichen Natur, Berlin.

Popper, Karl R. (1934 [1994]): Logik der Forschung, 10. Auflage, Tübingen.

Popper, Karl R. (1944 [1987]): Das Elend des Historizismus, 6. Auflage, Tübingen.

Popper, Karl R. (1945a [1992a]): Die offene Gesellschaft und ihre Feinde, Bd. I: Der Zauber Platons, 7. Auflage, Tübingen.

Popper, Karl R. (1945b [1992b]): Die offene Gesellschaft und ihre Feinde, Bd. II: Falsche Propheten: Hegel, Marx und die Folgen, 7. Auflage, Tübingen. 
Popper, Karl R. (1963 [1994]): Vermutungen und Widerlegungen. Das Wachstum der wissenschaftlichen Erkenntnis, 2 Teilbände, Tübingen.

Popper, Karl R. (1985 [1995]): The Rationality Principle, in: David Miller (Hrsg.), Popper Selections, Princeton, S. 357-365.

Raub, Werner / Thomas Voss (1981): Individuelles Handeln und gesellschaftliche Folgen, Darmstadt.

Riker, William H. / Peter C. Ordeshook (1973): An Introduction to Positive Political Theory, Englewood Cliffs.

Rosa, Hartmut / David Strecker / Andrea Kottmann (2007): Soziologische Theorien, Konstanz.

Rosenberg, Alex (2006): Philosophy of Science. A Contemporary Introduction, 2. Auflage, New York.

Salmon, Wesley C. (2006): Four Decades of Scientific Explanation, Pittsburgh.

Schmid, Michael (2006): Die Logik mechanismischer Erklärungen, Wiesbaden.

Simon, Herbert A. (1963): Problems of Methodology - Discussion, in: American Economic Review: Papers \& Proceedings 53, S. 229-231.

Simon, Herbert A. (1990): Bounded Rationality, in: John Eatwell / Murray Milgate / Peter Newman (Hrsg.), The New Palgrave: Utility and Probability, New York, S. 15-18.

Sober, Elliott (1983): Equilibrium Explanations, in: Philosophical Studies 43, S. 201-210.

Stegmüller, Wolfgang (1980): Neue Wege der Wissenschaftsphilosophie, Berlin - Heidelberg - New York.

Turner, Jonathan H. (1991): The Structure of Sociological Theory, 5. Auflage, Belmont.

Vanberg, Viktor (1975): Die zwei Soziologien: Individualismus und Kollektivismus in der Sozialtheorie, Tübingen.

Vollmer, Gerhard (1975): Evolutionäre Erkenntnistheorie, Stuttgart.

Wippler, Reinhard / Siegwart Lindenberg (1987): Collective Phenomena and Rational Choice, in: Jeffrey C. Alexander / Bernhard Giesen / Richard Münch / Neil J. Smelser (Hrsg.), The Micro-Macro Link, Berkeley, S. 135-152.

Wolf, Christof (1996): Gleich und gleich gesellt sich, Hamburg.

Woodward, James (2003): Making Things Happen. A Theory of Causal Explanation, Oxford.

Prof. Norman Braun Ph.D.

Ludwig-Maximilians-Universität München

Institut für Soziologie

Konradstr. 6

80801 München

Norman.Braun@soziologie.uni-muenchen.de 